 \\ Ophthalmology and Clinical Research
}

\section{A Tricky Case of Refractive Disorder in a Female Teenager}

Daniel Roquelaure and Georgios D Panos*

Department of Ophthalmology, Geneva University Hospitals, Geneva, Switzerland

\begin{abstract}
We present a tricky case of a teenage female patient who has been initially misdiagnosed as a case of optic neuropathy. A detailed examination including cycloplegic refraction revealed that the patient presented accommodative disorders because of latent hyperopia and previous stressful situation.

Every young patient coming in Ophthalmology service complaining for blurred vision, regardless of his age, or ocular past, need to have a cycloplegic objective refraction.

Keywords: Accommodative disorders, Cycloplegia, Optic neuropathy, Refractive errors
\end{abstract}

\section{Introduction}

Refractive disorders are a common condition found in the children. Fulton et al., [1] described a rate of $19 \%$ astigmatism of normal infants, at least twice the incidence in adults. Female sex, higher grade level and using computers regularly were significantly associated with refractive error according to Sewunet et al., [2].

For undiagnosed patients, who compensated during childhood, it can be made manifest in an acute way during adolescence. Accuracy of accommodation is reduced in uncorrected children with high astigmatism and high hyperopia [3]. Then the complaints can be very large and it becomes tricky for the clinician to make the diagnosis.

\section{Case Report}

In our center, a 14-year-old girl described decrease of visual acuity in the right eye since 3 weeks, without any pain or redness. She did a major depressive disorder leading to hospitalization 2 months before, and was under citalopram for a short period. Except that, she had no other past medical or ocular history.

On examination, best-corrected visual acuity was 0.3 decimal Snellen units in the right eye $(+0.25 / 0 / 0)$ and 0.8 decimal Snellen units

*Corresponding author: Georgios Panos, Department of Ophthalmology, Geneva University Hospitals, Alcide-Jentzer 22, CH 1211, Geneva, Switzerland, Tel: +41 767459028; Fax: +41 223828382; E-mail: gdpanos@gmail.com

Citation: Roquelaure D, Panos GD (2015) A Tricky Case of Refractive Disorder in a Female Teenager. J Ophthalmic Clin Res 2: 010.

Received: November 25, 2014; Accepted: April 13, 2015; Published: April 27, 2015 in the left eye $(+0.25 /+0.25 / 65)$. Pupils were isocore, normoreactive, with no relative afferent pupillary defect. Anterior segment examination was unremarkable in both eyes. Fundus examination was also normal, without any optic nerve abnormality.

The Lang stereo test II score was $2 / 4$ (200moa). The Hardy-Rand-Rittler color vision test score was 21/24 in the right eye, $24 / 24$ in the left eye.

Visual field (Octopus System) revealed numerous peripheral absolute defects in every quadrant of the right eye (Figure 1), with a relative good respect of the macular area; no defect was found in the left eye. Answers of visual evoked potentials were normal and symmetrical, for monocular and binocular vision. An optical coherence tomography (Heidelberg Engineering) was also normal both eyes.
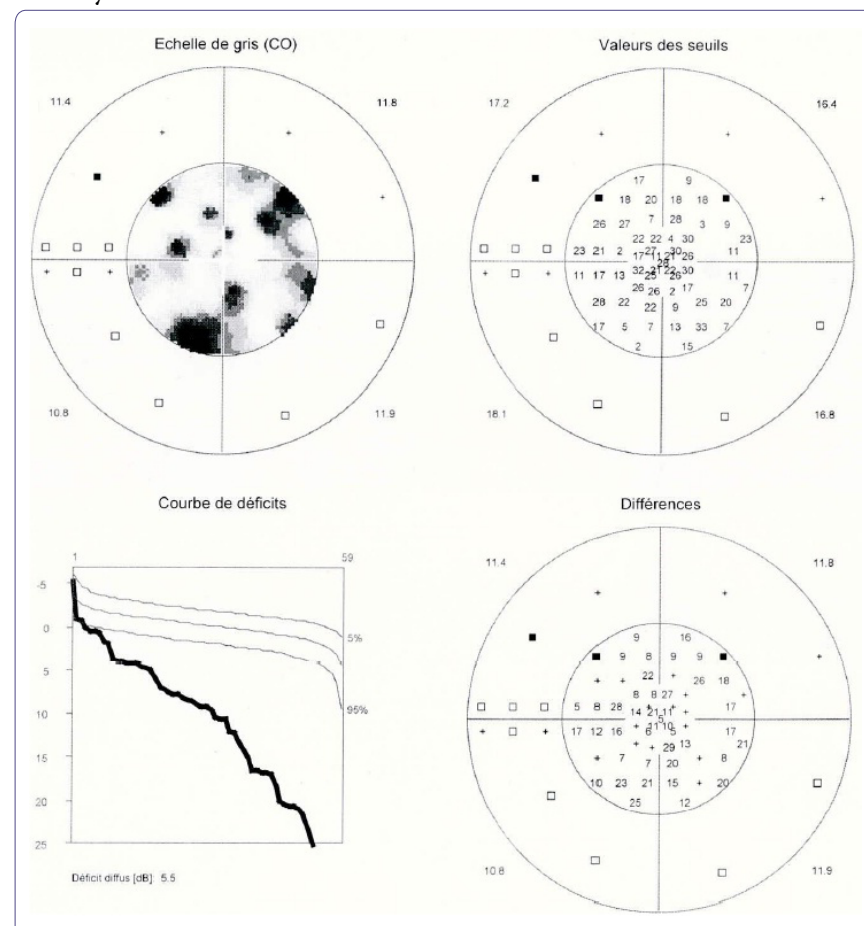

Figure 1: The first visual field examination revealed numerous peripheral absolute defects in every quadrant of the right eye.

Considering the possible diagnosis of right optic nerve lesion, like retrobulbar neuritis, brain tumour, or multiple sclerosis, we organized an urgent cerebral and orbit MRI with contrast product Gadolinium. It didn't show any abnormality at last.

Ten days later, we found a better best-corrected visual acuity at 0.8 decimal Snellen units both eyes, with a different refraction (OD -1), and Lang stereotest I was 3/3 (1200 moa). The rest of the examination was without any particularity. These last results tend to conclude to a refractive and accommodative disorder.

Consequently, we organized a cycloplegia, according to the protocol following: one drop of oxybuprocaine, and then three drops of cyclopentolate $0.5 \%$ every 10 minutes. After that, we did an 
objective refraction with autorefractometer (Retinomax) and manual skiascopy.

The visual acuity with the correction of cycloplegic refraction was 1.0 decimal Snellen units in both eyes $(\mathrm{OD}+1 /+0.5 / 70$, OS $+1.5 /+0.25 / 65)$. Five days later the visual field examination and visual evoked potentials were within normal limits (Figure 2).

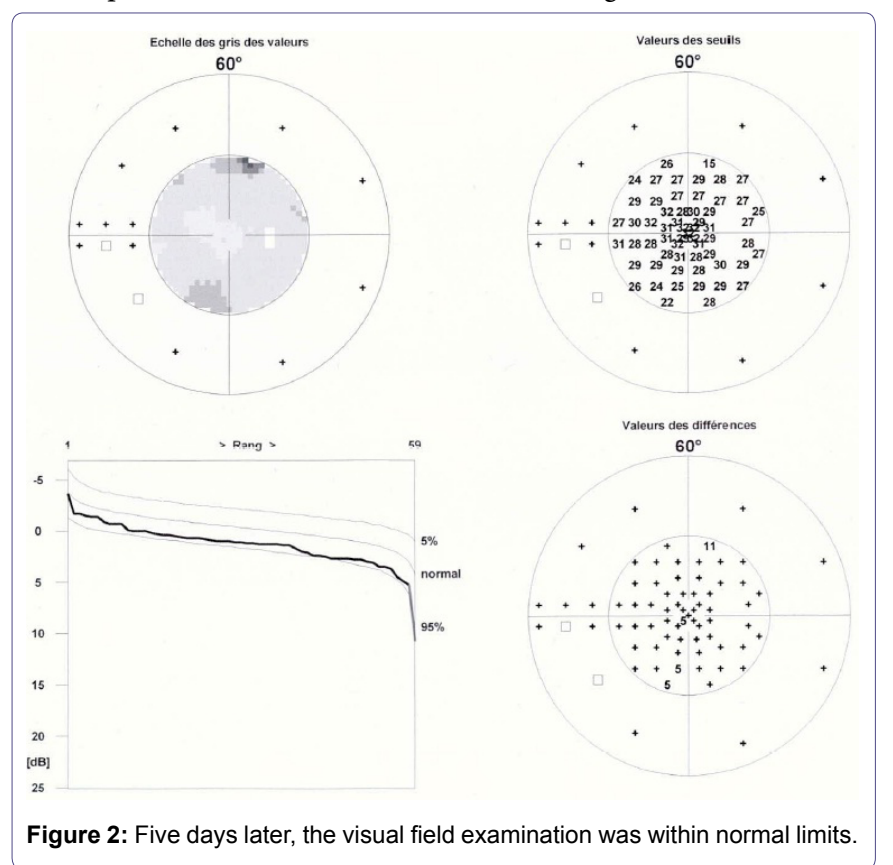

Finally diagnosis was hypermetropia, astigmatism, and accomodative disorders. So the problem was resolved by a prescription of adapted glasses (OD $+0.50 /+0.50 / 70$, OS $0.75 /+0.25 / 65)$.

\section{Discussion}

Refractive disorders can cause many complaints, overall on a teenager. Visual field defects or color vision abnormalities with poor visual acuity can be misdiagnosed like optic neuritis [4].

Fulton and al [1] explained that more than one-half of astigmatic infants were screened only with cycloplegia. Even if atropine is the gold standard, the cyclopentolate-tropicamide combination is satisfactory for routine cycloplegia in children [5,6]. Atropine causes indeed prolonged blurred vision contrary to the cyclopentolate, and could potentially have a disastrous effect on the development of vision in the youngest patients [7].
To take the most possible adapted refractive measures, there are a lot of machines. Here we use Retinomax, known for diagnosing abnormal astigmatism ( $>$ or $=2 \mathrm{D}$ ) with $51 \%$ to $84 \%$ sensitivity rates and $98 \%$ to $90 \%$ specificity rates, depending on the chosen threshold of manifest astigmatism [8]. Moreover, this tool does easy feasible measurements in very young children thanks to quick mode, with no significant difference between the quick one and the normal one in measuring manifest refraction.

\section{Conclusion}

To conclude, every young patient coming in Ophthalmology service complaining for blurred vision, regardless of his age, or ocular past, need to have a cycloplegic objective refraction. Refractive disorders are indeed potentially discomfortable for the patient, misdiagnosed by the Ophthalmologist, whereas they are easy to detect and treat.

\section{References}

1. Fulton AB, Dobson V, Salem D, Mar C, Petersen RA, et al. (1980) Cycloplegic refractions in infants and young children. Am J Ophthalmol 90: 239-247.

2. Sewunet SA, Aredo KK, Gedefew M (2014) Uncorrected refractive error and associated factors among primary school children in Debre Markos District, Northwest Ethiopia. BMC Ophthalmol 14: 95.

3. Harvey EM, Miller JM, Apple HP, Parashar P, Twelker JD, et al. (2014) Accommodation in astigmatic children during visual task performance. Invest Ophthalmol Vis Sci 55: 5420-5430.

4. Pérez-Cambrodí RJ, Gómez-Hurtado Cubillana A, Merino-Suárez ML, Piñero-Llorens DP, Laria-Ochaita C (2014) Optic neuritis in pediatric population: a review in current tendencies of diagnosis and management. J Optom 7: 125-130.

5. Celebi S, Aykan U (1999) The comparison of cyclopentolate and atropine in patients with refractive accommodative esotropia by means of retinoscopy, autorefractometry and biometric lens thickness. Acta Ophthalmol Scand 77: 426-429.

6. Alimgil ML, Erda N (1992) The cycloplegic effect of atropine in comparison with the cyclopentolate-tropicamide-phenylephrine combination. Klin Monbl Augenheilkd 201: 9-11.

7. Ingram RM, Barr A (1979) Refraction of 1-year-old children after cycloplegia with $1 \%$ cyclopentolate: comparison with findings after atropinisation. $\mathrm{Br} \mathrm{J}$ Ophthalmol 63: 348-352.

8. Cordonnier M, Dramaix M (1999) Screening for refractive errors in children: accuracy of the hand held refractor Retinomax to screen for astigmatism. $\mathrm{Br}$ J Ophthalmol 83: 157-61. 